\title{
The dominant eigenvalue of an essentially nonnegative tensor
}

\author{
L. P. Zhang ${ }^{1, * \dagger}$, L. Q. Qi ${ }^{2}$, Z. Y. Luo ${ }^{3}$ and Y. $\mathrm{Xu}^{2}$ \\ ${ }^{1}$ Department of Mathematical Sciences, Tsinghua University, Beijing, China \\ ${ }^{2}$ Department of Applied Mathematics, The Hong Kong Polytechnic University, Hung Hom, Kowloon, Hong Kong \\ ${ }^{3}$ State Key Laboratory of Rail Traffic Control and Safety, Beijing Jiaotong University, Beijing, China
}

\begin{abstract}
SUMMARY
It is well known that the dominant eigenvalue of a real essentially nonnegative matrix is a convex function of its diagonal entries. This convexity is of practical importance in population biology, graph theory, demography, analytic hierarchy process, and so on. In this paper, the concept of essentially nonnegativity is extended from matrices to higher-order tensors, and the convexity and log convexity of dominant eigenvalues for such a class of tensors are established. Particularly, for any nonnegative tensor, the spectral radius turns out to be the dominant eigenvalue and hence possesses these convexities. Finally, an algorithm is given to calculate the dominant eigenvalue, and numerical results are reported to show the effectiveness of the proposed algorithm. Copyright (c) 2013 John Wiley \& Sons, Ltd.
\end{abstract}

Received 26 December 2011; Revised 22 January 2013; Accepted 18 February 2013

KEY WORDS: essentially nonnegative tensor; dominant eigenvalue; convex function; spectral radius; algorithm

\section{INTRODUCTION}

Tensors are increasingly ubiquitous in various areas of applied, computational, and industrial mathematics and have wide applications in data analysis and mining, information science, signal/image processing, computational biology, and so on; see the workshop report [1] and references therein. A tensor can be regarded as a higher-order generalization of a matrix, which takes the form

$$
\mathcal{A}=\left(A_{i_{1} \cdots i_{m}}\right), \quad A_{i_{1} \cdots i_{m}} \in R, \quad 1 \leqslant i_{1}, \ldots, i_{m} \leqslant n .
$$

Such a multi-array $\mathcal{A}$ is said to be an $m$-order $n$-dimensional square real tensor with $n^{m}$ entries $A_{i_{1} \cdots i_{m}}$. In this regard, a vector is a first-order tensor and a matrix is a second-order tensor. Tensors of order more than two are called higher-order tensors.

Analogous with that of matrices, the theory of eigenvalues and eigenvectors is one of the fundamental and essential components in tensor analysis. Seventy-two references on eigenvalues of tensors can be found in the bibliography [2]. A wide range of practical applications can be found the references there. Compared with that of matrices, eigenvalue problems for higher-order tensors are nonlinear because of their multilinear structure. Various types of eigenvalues are defined for higherorder tensors in the setting of multilinear algebra. For example, the eigenvalue, the $H$-eigenvalue, the $E$-eigenvalue, the $Z$-eigenvalue, the $N$-eigenvalue defined by Qi for even order symmetric tensors [3], the $l^{p}$ eigenvalues for general order symmetric tensors, and the mode- $i$ eigenvalues for general square tensors defined by $\operatorname{Lim}[4]$, the $M$-eigenvalue for a partially symmetric fourth-order tensor, defined by Qi et al. [5], the $D$-eigenvalue for a fourth-order symmetric tensor and a secondorder symmetric tensor, defined by Qi et al. [6], eigenvalues of general square tensors extended by

\footnotetext{
*Correspondence to: L. P. Zhang, Department of Mathematical Sciences, Tsinghua University, Beijing, China.

$\dagger$ E-mail: lzhang@math.tsinghua.edu.cn
} 
Qi in [2], Chang et al. in [7], and equivalent eigenvalue pair classes by Cartwright and Sturmfels [8]. Here, we are concerned with the one in $[2,7]$ as reviewed in the succeeding texts.

Definition 1.1

Let $\mathrm{C}$ be the complex field. For a vector $x \in \mathrm{C}^{n}$, we use $x_{i}$ to denote its components and $x^{[m-1]}$ to denote a vector in $\mathrm{C}^{n}$ such that

$$
x_{i}^{[m-1]}=x_{i}^{m-1}
$$

for all $i . \mathcal{A} x^{m-1}$ denotes a vector in $\mathrm{C}^{n}$, whose $i$ th component is

$$
\sum_{i_{2}, \ldots, i_{m}=1}^{n} A_{i i_{2} \cdots i_{m}} x_{i_{2}} \cdots x_{i_{m}}
$$

A pair $(\lambda, x) \in \mathrm{C} \times\left(\mathrm{C}^{n} \backslash\{0\}\right)$ is called an eigenvalue-eigenvector pair of $\mathcal{A}$, if they satisfy

$$
\mathcal{A} x^{m-1}=\lambda x^{[m-1]} .
$$

Nonnegative tensors, arising from multilinear pagerank [4], spectral hypergraph theory [9-11], and higher-order Markov chains [12], and so on form a singularly important class of tensors and have attracted more and more attention because they share some intrinsic properties with those of the nonnegative matrices. One of those properties is the Perron-Frobenius theorem on eigenvalues. In [13], Chang et al. generalized the Perron-Frobenius theorem for nonnegative matrices to irreducible nonnegative tensors. In [14], Friedland et al. generalized the Perron-Frobenius theorem to weakly irreducible nonnegative tensors. Further generalization of the Perron-Frobenius theorem to nonnegative tensors can be found in [15]. Numerical methods for finding the spectral radius of nonnegative tensors are subsequently proposed. $\mathrm{Ng}$ et al. [12] provided an iterative method to find the largest eigenvalue of an irreducible nonnegative tensor by extending the Collatz method [16] for calculating the spectral radius of an irreducible nonnegative matrix. The Ng-Qi-Zhou method is efficient, but it is not always convergent for irreducible nonnegative tensors. Chang et al. [17] extended the notion of primitive matrices into the realm of tensors and established the convergence of the Ng-Qi-Zhou method for primitive tensors. Zhang and Qi [18] established global linear convergence of the Ng-Qi-Zhou method for essentially positive tensors. Liu et al. [19] proposed an always convergent algorithm for computing the largest eigenvalue of an irreducible nonnegative tensors. Zhang et al. [20] established its explicit linear convergence rate for weakly positive tensors.

The essentially nonnegative tensor we defined in this paper is ultimately related to the nonnegative tensor and includes the latter one as a special case. It is a higher-order generalization of the so-called essentially nonnegative matrix, whose off-diagonal entries are all nonnegative. Such a class of matrices possesses nice properties on eigenvalues. It follows from the famous Perron-Frobenius theorem for nonnegative matrices that for any essentially nonnegative matrix $A$, there exists a real eigenvalue with a nonnegative eigenvector, which is the largest one among real parts of all other eigenvalues of $A$. This special eigenvalue, termed as $r(A)$, is often called the dominant eigenvalue of $A$. Moreover, $r(A)$ is known as a convex function of the diagonal entries of $A$. This convexity is a fundamental property for essentially nonnegative matrices [21-23] and has numerous applications, not only in many branches of mathematics, such as graph theory [24] and differential equations [23], but also in practical fields, for example, population biology [23] and analytic hierarchy process [25], as well. A natural question arises: Does this convexity maintain for higher-order essentially nonnegative tensors? In this paper, we will give an affirmative answer to this question.

Similar to the essentially nonnegative matrix, an essentially nonnegative tensor has a real eigenvalue with the property that it is greater than or equal to the real part of every eigenvalue of $\mathcal{A}$. We also call it the dominant eigenvalue of $\mathcal{A}$ and denoted by $\lambda(\mathcal{A})$. Particularly, if $\mathcal{A}$ is nonnegative, we have $\rho(\mathcal{A})=\lambda(\mathcal{A})$, where $\rho(\mathcal{A})$ is the spectral radius of $\mathcal{A}$. By employing the technique proposed in [23], we manage to obtain that the dominant eigenvalue is a convex function of the diagonal elements for any essentially nonnegative tensor. In addition, it is also a convex function of all elements of a tensor in some special convex set of tensors. Furthermore, the log convexity is also exploited for 
essentially nonnegative tensors with whose entries are either identically zero or log convex of some real univariate functions. Finally, we propose an algorithm to calculate the dominant eigenvalue, convergence of the proposed algorithm is established, and numerical results are reported to show the effectiveness of the proposed algorithm.

This paper is organized as follows. In Section 2, we recall some preliminary results, introduce the concept of essentially nonnegative tensors, and characterize some basic properties of such tensors. In Section 3, we show that the spectral radius of nonnegative tensors is a convex function of the diagonal elements and so is the dominant eigenvalue of essentially nonnegative tensors. Section 4 is devoted to the log convexity of the dominant eigenvalue. In Section 5, we give an algorithm to calculate the dominant eigenvalue, and some numerical results are reported. An application and some concluding remarks are made in Section 6.

\section{PRELIMINARIES AND ESSENTIALLY NONNEGATIVE TENSORS}

We start this section with some fundamental notions and properties on tensors. An $m$-order $n$ dimensional tensor $\mathcal{A}$ is called nonnegative (or, respectively, positive) if $A_{i_{1} \cdots i_{m}} \geqslant 0$ (or, respectively, $A_{i_{1} \cdots i_{m}}>0$ ). The $m$-order $n$-dimensional unit tensor, denoted by $\mathcal{I}$, is the tensor whose entries are $\delta_{i_{1} \ldots i_{m}}$ with $\delta_{i_{1} \ldots i_{m}}=1$ if and only if $i_{1}=\cdots=i_{m}$ and otherwise zero. The symbol $\mathcal{A} \geqslant \mathcal{B}$ means that $\mathcal{A}-\mathcal{B}$ is a nonnegative tensor. A tensor $\mathcal{A}$ is called reducible, if there exists a nonempty proper index subset $I \subset\{1,2, \ldots, n\}$ such that

$$
A_{i_{1} \cdots i_{m}}=0, \quad \forall i_{1} \in I, \quad \forall i_{2}, \ldots, i_{m} \notin I .
$$

Otherwise, we say $\mathcal{A}$ is irreducible. We call $\rho(\mathcal{A})$ the spectral radius of tensor $\mathcal{A}$ if

$$
\rho(\mathcal{A})=\max \{|\lambda|: \lambda \text { is an eigenvalue of } \mathcal{A}\}
$$

where $|\lambda|$ denotes the modulus of $\lambda$. An immediate consequence on the spectral radius follows directly from Corollary 3 in [3].

\section{Lemma 2.1}

Let $\mathcal{A}$ be an $m$-order $n$-dimensional tensor. Suppose that $\mathcal{B}=a(\mathcal{A}+b \mathcal{I})$, where $a$ and $b$ are two real numbers. Then $\mu$ is an eigenvalue of $\mathcal{B}$ if and only if $\mu=a(\lambda+b)$ and $\lambda$ is an eigenvalue of $\mathcal{A}$. In this case, they have the same eigenvectors. Moreover, $\rho(\mathcal{B}) \leqslant|a|(\rho(\mathcal{A})+|b|)$.

Let $P:=\left\{x \in \mathbf{R}^{n}: x_{i} \geqslant 0,1 \leqslant i \leqslant n\right\}$, and $\operatorname{int}(P)=\left\{x \in \mathbf{R}^{n}: x_{i}>0,1 \leqslant i \leqslant n\right\}$. The Perron-Frobenius theorem for nonnegative tensors is as discussed in the succeeding texts, following by [13, Theorem 1.4].

\section{Theorem 2.1}

If $\mathcal{A}$ is an irreducible nonnegative tensor of order $m$ and dimension $n$, then there exist $\lambda_{0}>0$ and $x_{0} \in \operatorname{int}(P)$ such that

$$
\mathcal{A} x_{0}^{m-1}=\lambda_{0} x_{0}^{[m-1]} .
$$

Moreover, if $\lambda$ is an eigenvalue with a nonnegative eigenvector, then $\lambda=\lambda_{0}$. If $\lambda$ is an eigenvalue of $\mathcal{A}$, then $|\lambda| \leqslant \lambda_{0}$.

The well-known Collatz minimax theorem [16] for irreducible nonnegative matrices has been extended to irreducible nonnegative tensors in [13, Theorem 4.2]. 
Theorem 2.2

Assume that $\mathcal{A}$ is an irreducible nonnegative tensor of order $m$ dimension $n$. Then

$$
\min _{x \in \operatorname{int}(P)} \max _{x_{i}>0} \frac{\left(\mathcal{A} x^{m-1}\right)_{i}}{x_{i}^{m-1}}=\lambda_{0}=\max _{x \in \operatorname{int}(P)} \min _{x_{i}>0} \frac{\left(\mathcal{A} x^{m-1}\right)_{i}}{x_{i}^{m-1}},
$$

where $\lambda_{0}$ is the unique positive eigenvalue corresponding to a positive eigenvector.

For nonnegative tensors, Yang and Yang [15] asserted that the spectral radius is an eigenvalue, which is a generalization of the weak Perron-Frobenius theorem for nonnegative matrices. We state it [15, Theorem 2.3 and Lemma 5.8] in the following theorem.

\section{Theorem 2.3}

Assume that $\mathcal{A}$ is a nonnegative tensor of order $m$ dimension $n$, then $\rho(\mathcal{A})$ is an eigenvalue of $\mathcal{A}$ with a nonzero nonnegative eigenvector. Moreover, for any $x \in \operatorname{int}(P)$, we have

$$
\min _{1 \leqslant i \leqslant n} \frac{\left(\mathcal{A} x^{m-1}\right)_{i}}{x_{i}^{m-1}} \leqslant \rho(\mathcal{A}) \leqslant \max _{1 \leqslant i \leqslant n} \frac{\left(\mathcal{A} x^{m-1}\right)_{i}}{x_{i}^{m-1}} .
$$

The following inequality and continuity of the spectral radius were given in [15, Lemma 3.5] and the proof of [15, Theorem2.3], respectively.

Lemma 2.2

Let $\mathcal{A}$ be a nonnegative tensor of order $m$ and dimension $n$, and $\varepsilon>0$ be a sufficiently small number. Suppose $\mathcal{A} \leqslant \mathcal{B}$, then $\rho(\mathcal{A}) \leqslant \rho(\mathcal{B})$. Furthermore, if $\mathcal{A}_{\varepsilon}=\mathcal{A}+\mathcal{E}$ where $\mathcal{E}$ denotes the tensor with every entry being $\varepsilon$, then

$$
\lim _{\varepsilon \rightarrow 0} \rho\left(\mathcal{A}_{\varepsilon}\right)=\rho(\mathcal{A})
$$

On the basis of the preceding results, we can easily obtain the following lemma.

Lemma 2.3

Suppose that $\mathcal{A}$ is an irreducible nonnegative tensor of order $m$ dimension $n$ and that there exists a nonzero vector $x \in P$ and a real number $\beta$ such that

$$
\mathcal{A} x^{m-1} \leqslant \beta x^{[m-1]} .
$$

Then $\beta>0, x \in \operatorname{int}(P)$, and $\rho(\mathcal{A}) \leqslant \beta$. Furthermore, $\rho(\mathcal{A})<\beta$ unless equality holds in (2).

Proof

Assume on the contrary that for $x \in \operatorname{int}(P)$ there exists a nonempty proper index subset $I \subset$ $\{1,2, \ldots, n\}$ such that $x_{i}=0$ for $i \in I$ and $x_{i}>0$ for $i \notin I$. It follows from (2) that

$$
A_{i_{1} \cdots i_{m}}=0, \quad \forall i_{1} \in I, \quad \forall i_{2}, \ldots, i_{m} \notin I .
$$

A contradiction to the irreducibility of $\mathcal{A}$ comes, which henceforth implies that $x \in \operatorname{int}(P)$. Together with Lemma 2.2 in [12], $\mathcal{A} x^{m-1} \in \operatorname{int}(P)$ is established. It further deduces that $\beta>0$, and then the last statement holds from Lemma 5.9 in [15]. This completes the proof.

A simple but useful result follows immediately from Lemmas 2.2 and 2.3.

Lemma 2.4

Let $\mathcal{A}$ and $\mathcal{B}$ be irreducible nonnegative tensors of order $m$ dimension $n$. If $\mathcal{A} \leqslant \mathcal{B}$ and $\mathcal{A} \neq \mathcal{B}$, then $\rho(\mathcal{A})<\rho(\mathcal{B})$. 
Proof

By Lemma 2.2, $\rho(\mathcal{A}) \leqslant \rho(\mathcal{B})$. As $\mathcal{B}$ is irreducible, Theorem 2.1 implies that there exists $x \in \operatorname{int}(P)$ such that

$$
\mathcal{A} x^{m-1} \leqslant \mathcal{B} x^{m-1}=\rho(\mathcal{B}) x^{[m-1]} .
$$

As $x \in \operatorname{int}(P)$ and $\mathcal{A} \neq \mathcal{B}$, equality cannot hold in (3). The desired strict inequality $\rho(\mathcal{A})<\rho(\mathcal{B})$ holds from Lemma 2.3.

The remainder of this section is devoted to the essentially nonnegative tensor, with the introduction of its definition and some basic properties.

\section{Definition 2.1}

Let $\mathcal{A}$ be an $m$-order and $n$-dimensional tensor. $\mathcal{A}$ is said to be essentially nonnegative if all its off-diagonal entries are nonnegative.

\section{Theorem 2.4}

Let $\mathcal{A}$ be an $m$-order and $n$-dimensional essentially nonnegative tensor. Then there exists $\alpha>0$ such that $\alpha \mathcal{I}+\mathcal{A}$ is nonnegative. Moreover, $\mathcal{A}$ has a real eigenvalue $\lambda(\mathcal{A})$ with corresponding eigenvector in $P$ and $\lambda(\mathcal{A}) \geqslant \operatorname{Re} \lambda$ for every eigenvalue $\lambda$ of $\mathcal{A}$. Furthermore,

$$
\lambda(\mathcal{A})=\rho(\alpha \mathcal{I}+\mathcal{A})-\alpha .
$$

Proof

Take

$$
\alpha=\max _{1 \leqslant i \leqslant n}\left|A_{i \ldots i}\right|+1 .
$$

Clearly, $\alpha>0$, and $\alpha \mathcal{I}+\mathcal{A}$ is nonnegative. By Lemma 2.1 and Theorem 2.3, we have

$$
\rho(\alpha \mathcal{I}+\mathcal{A})=\alpha+\lambda_{1},
$$

where $\lambda_{1}$ is an eigenvalue of $\mathcal{A}$ with corresponding eigenvector in $P$. Thus, (4) implies $\lambda_{1} \in \mathrm{R}$. Let $\lambda(\mathcal{A})=\lambda_{1}$, It follows from Lemma 2.1 that

$$
\begin{aligned}
\lambda(\mathcal{A})+\alpha & =\max \{|\alpha+\lambda|: \lambda \text { is an eigenvalue of } \mathcal{A}\} \\
& \geqslant|\alpha+\lambda| \geqslant \alpha+\operatorname{Re} \lambda .
\end{aligned}
$$

The desired result arrives.

We call such an eigenvalue in the preceding theorem the dominant eigenvalue of $\mathcal{A}$. Throughout this paper, $\rho(\mathcal{A})$ and $\lambda(\mathcal{A})$ will denote the spectral radius and dominant eigenvalue, respectively, of a tensor $\mathcal{A}$. In the next section, we will show that both $\rho(\mathcal{A})$ and $\lambda(\mathcal{A})$ are convex functions of the diagonal elements of $\mathcal{A}$.

\section{CONVEXITY OF THE SPECTRAL RADIUS AND THE DOMINANT EIGENVALUE}

On the basis of Theorems 2.1 and 2.3, we proceed with the convexity of the dominant eigenvalue of essentially nonnegative tensors in this section. It can be verified that the diagonal entries have nothing to do with the irreducibility of a tensor. Specifically, let $\mathcal{A}$ be an essentially nonnegative tensor of order $m$ and dimension $n$, define a nonnegative tensor $\mathcal{B}$ by $B_{i_{1} \ldots i_{m}}=0$ if $i_{1}=\cdots=i_{m}$, and the others are $A_{i_{1} \ldots i_{m}}$. Then $\mathcal{A}$ is irreducible if and only if $\mathcal{B}$ is. Equivalently, $\mathcal{A}$ is irreducible if and only if $\mathcal{A}+\alpha \mathcal{I}$ is, whenever it is nonnegative. Thus, by Lemma 2.2 and Theorem 2.4, it is sufficient to consider the class of irreducible nonnegative tensors. 


\section{Theorem 3.1}

If $\mathcal{A}$ is a given irreducible nonnegative tensor of order $m$ and dimension $n$, and $\mathcal{D}$ is allowed to vary in the class of nonnegative diagonal tensors, then the spectral radius $\rho(\mathcal{A}+\mathcal{D})$ is a convex function of the diagonal entries of $\mathcal{D}$. That is, for nonnegative diagonal tensors $\mathcal{C}$ and $\mathcal{D}$, we have

$$
\rho(\mathcal{A}+t \mathcal{C}+(1-t) \mathcal{D}) \leqslant t \rho(\mathcal{A}+\mathcal{C})+(1-t) \rho(\mathcal{A}+\mathcal{D}), \quad \forall t \in[0,1] .
$$

Moreover, equality holds in (5) for some $t \in(0,1)$ if and only if $\mathcal{D}-\mathcal{C}$ is a scalar multiple of the unit tensor $\mathcal{I}$.

Proof

As both $\mathcal{A}+\mathcal{C}$ and $\mathcal{A}+\mathcal{D}$ are irreducible nonnegative tensors, by Theorems 2.1 and 2.3 , we have $\rho(\mathcal{A}+\mathcal{C})>0, \rho(\mathcal{A}+\mathcal{D})>0$, and there exist $x, y \in \operatorname{int}(P)$ such that

$$
(\mathcal{A}+\mathcal{C}) x^{m-1}=\rho(\mathcal{A}+\mathcal{C}) x^{[m-1]}, \quad(\mathcal{A}+\mathcal{D}) y^{m-1}=\rho(\mathcal{A}+\mathcal{D}) y^{[m-1]} .
$$

That is, for $i=1,2, \ldots, n$, we have

$$
\begin{aligned}
\rho(\mathcal{A}+\mathcal{C}) & =C_{i \ldots i}+\sum_{i_{2} \ldots i_{m}=1}^{n} A_{i i_{2} \ldots i_{m}} \frac{x_{i_{2}} \cdots x_{i_{m}}}{x_{i}}, \\
\rho(\mathcal{A}+\mathcal{D}) & =D_{i \ldots i}+\sum_{i_{2} \ldots i_{m}=1}^{n} A_{i i_{2} \ldots i_{m}} \frac{y_{i_{2}} \cdots y_{i_{m}}}{y_{i}},
\end{aligned}
$$

and hence $\rho(\mathcal{A}+\mathcal{C})-C_{i \ldots i}>0$ and $\rho(\mathcal{A}+\mathcal{D})-D_{i \ldots i}>0$. The inequality between geometric and arithmetic means yields

$$
\begin{aligned}
\left(\sum_{i_{2} \ldots i_{m}=1}^{n} A_{i i_{2} \ldots i_{m}} \frac{x_{i_{2}} \cdots x_{i_{m}}}{x_{i}}\right)^{t}\left(\sum_{i_{2} \ldots i_{m}=1}^{n} A_{i i_{2} \ldots i_{m}} \frac{y_{i_{2}} \cdots y_{i_{m}}}{y_{i}}\right)^{1-t} \leqslant & t\left(\rho(\mathcal{A}+\mathcal{C})-C_{i \ldots i}\right) \\
& +(1-t)\left(\rho(\mathcal{A}+\mathcal{D})-D_{i \ldots i}\right) .
\end{aligned}
$$

Therefore, Hölder's inequality and Theorem 2.2 give from (6)

$$
\begin{aligned}
\rho(\mathcal{A}+t \mathcal{C}+(1-t) \mathcal{D}) & \leqslant \max _{1 \leqslant i \leqslant n}\left\{t C_{i \ldots i}+(1-t) D_{i \ldots i}+\sum_{i_{2} \ldots i_{m}=1}^{n} A_{i i_{2} \ldots i_{m}} \frac{z_{i_{2}} \cdots z_{i_{m}}}{z_{i}}\right\} \\
& \leqslant t \rho(\mathcal{A}+\mathcal{C})+(1-t) \rho(\mathcal{A}+\mathcal{D}),
\end{aligned}
$$

where $z_{i}=x_{i}^{t} y_{i}^{1-t}$ for $i=1, \ldots, n$. This shows that (5) holds.

The inequality between geometric and arithmetic means implies that equality in (5) holds for $t \in(0,1)$ if and only if $\rho(\mathcal{A}+\mathcal{C})-C_{i \ldots i}=\rho(\mathcal{A}+\mathcal{D})-D_{i \ldots i}$ for $i=1, \ldots, n$, that is, $\mathcal{D}-\mathcal{C}=\gamma \mathcal{I}$, where $\gamma=\rho(\mathcal{A}+\mathcal{D})-\rho(\mathcal{A}+\mathcal{C})$. This completes the proof.

The convexity involved in Theorem 3.1 can be extended to the case of essentially nonnegative tensors as follows.

\section{Corollary 3.1}

If $\mathcal{A}$ is a given irreducible essentially nonnegative tensor of order $m$ dimension $n$ and $\mathcal{D}$ is allowed to vary in the class of diagonal tensors, then the dominant eigenvalue $\lambda(\mathcal{A}+\mathcal{D})$ is a convex function of the diagonal entries of $\mathcal{D}$. That is, for diagonal tensors $\mathcal{C}$ and $\mathcal{D}$, we have

$$
\lambda(\mathcal{A}+t \mathcal{C}+(1-t) \mathcal{D}) \leqslant t \lambda(\mathcal{A}+\mathcal{C})+(1-t) \lambda(\mathcal{A}+\mathcal{D}), \quad \forall t \in[0,1] .
$$

Moreover, equality holds in (7) for some $t \in(0,1)$ if and only if $\mathcal{D}-\mathcal{C}$ is a scalar multiple of the unit tensor $\mathcal{I}$. 
Proof

Take

$$
\alpha=1+\max _{1 \leqslant i \leqslant n}\left\{\left|A_{i \ldots i}\right|+\left|C_{i \ldots i}\right|+\left|D_{i \ldots i}\right|\right\} .
$$

Then $\alpha \mathcal{I}+\mathcal{A}+\mathcal{C}$ and $\alpha \mathcal{I}+\mathcal{A}+\mathcal{D}$ are all irreducible nonnegative tensors. By Theorems 2.4 and 3.1 , we have for $0 \leqslant t \leqslant 1$

$$
\begin{aligned}
\lambda(\mathcal{A}+t \mathcal{C}+(1-t) \mathcal{D})+\alpha & =\rho(\alpha \mathcal{I}+\mathcal{A}+t \mathcal{C}+(1-t) \mathcal{D}) \\
& \leqslant t \rho(\alpha \mathcal{I}+\mathcal{A}+\mathcal{C})+(1-t) \rho(\alpha \mathcal{I}+\mathcal{A}+\mathcal{D}) \\
& =t \lambda(\mathcal{A}+\mathcal{C})+(1-t) \lambda(\mathcal{A}+\mathcal{D})+\alpha,
\end{aligned}
$$

which yields (7). This completes the proof.

Invoking the continuity presented in Lemma 2.2, it is easy to see that Theorem 3.1 and Corollary 3.1 hold even when $\mathcal{A}$ is reducible. Moreover, Theorem 3.1 and Corollary 3.1 give necessary and sufficient conditions for the strict convexity. It is worth pointing out that the convexity of the dominant eigenvalue only works on the diagonal elements rather than on all elements of the essentially nonnegative tensor, except for some special cases. By collecting all symmetric essentially nonnegative tensors of order $m$ and dimension $n$, we can obtain a closed convex cone, say $\mathcal{S}(m, n)$. The dominant eigenvalue of any tensor in $\mathcal{S}(m, n)$ remains convex of all elements of the corresponding tensor in the domain $\mathcal{S}(m, n)$, as the following proposition shows.

Proposition 3.1

For any $\mathcal{A}, \mathcal{B} \in \mathcal{S}(m, n)$, and any $t \in[0,1]$, we have

$$
\lambda(t \mathcal{A}+(1-t) \mathcal{B}) \leqslant t \lambda(\mathcal{A})+(1-t) \lambda(\mathcal{B}) .
$$

Proof

For any $\mathcal{A}, \mathcal{B} \in \mathcal{S}(m, n)$, there exists an integer $k>0$ such that $\mathcal{A}+k \mathcal{I}$ and $\mathcal{B}+k \mathcal{I}$ are nonnegative and symmetric and hence for any of their convex combinations. The Perron-Frobenius theorem then ensures that $\rho(\mathcal{A}+k \mathcal{I}), \rho(\mathcal{B}+k \mathcal{I})$, and $\rho(t \mathcal{A}+(1-t) \mathcal{B}+k \mathcal{I})(t \in[0,1])$ all act as eigenvalues of the corresponding nonnegative symmetric tensor. By the variational approach, it follows that

$$
\begin{aligned}
& \rho(t \mathcal{A}+(1-t) \mathcal{B}+k \mathcal{I}) \\
= & \max \left\{(t \mathcal{A}+(1-t) \mathcal{B}+k \mathcal{I}) x^{m}: \sum_{i=1}^{n} x_{i}^{m}=1\right\} \\
\leqslant & t \max \left\{(\mathcal{A}+k \mathcal{I}) x^{m}: \sum_{i=1}^{n} x_{i}^{m}=1\right\}+(1-t) \max \left\{(\mathcal{B}+k \mathcal{I}) x^{m}: \sum_{i=1}^{n} x_{i}^{m}=1\right\} \\
= & t \rho(\mathcal{A}+k \mathcal{I})+(1-t) \rho(\mathcal{B}+k \mathcal{I}) .
\end{aligned}
$$

Combining with the fact that $\rho(\mathcal{A}+k \mathcal{I})=\lambda(\mathcal{A})+k$, the desired convexity follows.

\section{LOG CONVEXITY OF THE SPECTRAL RADIUS AND THE DOMINANT EIGENVALUE}

If a function $f(x)$ is positive on its domain and $\log f(x)$ is convex, then $f(x)$ is called log convex. It is known that the sum or product of log convex functions is also log convex. In this section, we extend Kingman's theorem [23] for matrices to tensors. Our motivation for the following proof comes from [23].

Theorem 4.1

For $t \in[0,1]$, assume that $\mathcal{F}(t)=\left(F_{i_{1} \ldots i_{m}}(t)\right)$ is an $m$-order $n$-dimensional irreducible nonnegative tensor, and suppose that for $1 \leqslant i_{1}, \ldots, i_{m} \leqslant n, F_{i_{1} \ldots i_{m}}(t)$ is either identically zero or positive and 
a log convex function of $t$. Then $\rho(\mathcal{F}(t))$ is a log convex function of $t$ for $t \in[0,1]$. That is, if $\mathcal{F}(0)=\mathcal{A}, \mathcal{F}(1)=\mathcal{B}$, and a nonnegative tensor $\mathcal{G}(t)=\left(A_{i_{1} \ldots i_{m}}^{1-t} B_{i_{1} \ldots i_{m}}^{t}\right)$, then

$$
\rho(\mathcal{F}(t)) \leqslant \rho(\mathcal{G}(t)) \leqslant \rho(\mathcal{A})^{1-t} \rho(\mathcal{B})^{t} .
$$

Moreover, the first equality occurs in (8) for some $t$ with $t \in(0,1)$ if and only if

$$
\mathcal{F}(t)=\mathcal{G}(t),
$$

and the second equality occurs in (8) for some $t$ with $t \in(0,1)$ if and only if there exists a constant $\sigma>0$ and a positive diagonal matrix $D=\operatorname{diag}\left(d_{1}, \ldots, d_{n}\right)$ such that

$$
\mathcal{B}=\sigma \mathcal{A} \cdot D^{-(m-1)} \cdot \overbrace{D \cdots D}^{m-1} \text { with } B_{i_{1} i_{2} \ldots i_{m}}=\sigma A_{i_{1} i_{2} \ldots i_{m}} d_{i_{1}}^{-(m-1)} d_{i_{2}} \cdots d_{i_{m}} .
$$

Proof

Clearly, $\mathcal{G}(0)=\mathcal{F}(0)=\mathcal{A}$ and $\mathcal{G}(1)=\mathcal{F}(1)=\mathcal{B}$. The log convexity assumption on $F_{i_{1} \ldots i_{m}}(t)$ implies that, for $t \in[0,1]$,

$$
\mathcal{F}(t) \leqslant \mathcal{G}(t)
$$

which, together with Lemma 2.2, implies

$$
\rho(\mathcal{F}(t)) \leqslant \rho(\mathcal{G}(t)) .
$$

As $\mathcal{F}(t)$ is irreducible, if equality holds in (9) for some $t_{0}$ with $0<t_{0}<1$, Lemma 2.4 implies that $\mathcal{F}\left(t_{0}\right)=\mathcal{G}\left(t_{0}\right)$.

As $\mathcal{F}(0)$ and $\mathcal{F}(1)$ are irreducible nonnegative, Theorem 2.1 shows that there exist $x, y \in \operatorname{int}(P)$ such that

$$
\mathcal{A} x^{m-1}=\rho(\mathcal{A}) x^{[m-1]}, \quad \mathcal{B} y^{m-1}=\rho(\mathcal{B}) y^{[m-1]} .
$$

For a fixed $t \in(0,1)$, define $z=x^{1-t} y^{t}$, that is, $z_{i}=x_{i}^{1-t} y_{i}^{t}$ for $1 \leqslant i \leqslant n$. Then the $i$ th component of $\mathcal{G}(t) z^{m-1}$ satisfies

$$
\left(\mathcal{G}(t) z^{m-1}\right)_{i}=\sum_{i_{2} \ldots i_{m}=1}^{n} A_{i i_{2} \ldots i_{m}}^{1-t} B_{i i_{2} \ldots i_{m}}^{t} z_{i_{2}} \cdots z_{i_{m}} .
$$

Hence, Hölder's inequality gives

$$
\begin{aligned}
\left(\mathcal{G}(t) z^{m-1}\right)_{i} & \leqslant\left(\sum_{i_{2} \ldots i_{m}=1}^{n} A_{i i_{2} \ldots i_{m}} x_{i_{2}} \cdots x_{i_{m}}\right)^{1-t}\left(\sum_{i_{2} \ldots i_{m}=1}^{n} B_{i i_{2} \ldots i_{m}} y_{i_{2}} \cdots y_{i_{m}}\right)^{t} \\
& =\rho(\mathcal{A})^{1-t} \rho(\mathcal{B})^{t} z_{i}^{m-1} .
\end{aligned}
$$

It follows from Lemma 2.3 and (10) that

$$
\rho(\mathcal{G}(t)) \leqslant \rho(\mathcal{A})^{1-t} \rho(\mathcal{B})^{t} .
$$

Furthermore, equality holds in (10) for some $t \in(0,1)$ if and only if, for $1 \leqslant i \leqslant n$,

$$
B_{i i_{2} \ldots i_{m}} y_{i_{2}} \cdots y_{i_{m}}=\sigma_{i} A_{i i_{2} \ldots i_{m}} x_{i_{2}} \cdots x_{i_{m}} .
$$

Summing (11) over $i_{2} \ldots i_{m}$ yields

$$
\rho(\mathcal{B}) y_{i}^{m-1}=\sigma_{i} \rho(\mathcal{A}) x_{i}^{m-1} .
$$

Take

$$
\sigma=\frac{\rho(\mathcal{B})}{\rho(\mathcal{A})}, \quad d_{i}=\frac{x_{i}}{y_{i}}
$$


Then combining (11) and (12), we obtain

$$
B_{i i_{2} \ldots i_{m}}=\sigma A_{i i_{2} \ldots i_{m}} d_{i}^{-(m-1)} d_{i_{2}} \cdots d_{i_{m}},
$$

that is,

$$
\mathcal{B}=\sigma \mathcal{A} \cdot D^{-(m-1)} \cdot \overbrace{D \cdots D}^{m-1} \text {. }
$$

This completes the proof.

By Theorems 2.3 and 2.4, the preceding theorem also holds for the dominant eigenvalue of $\mathcal{F}(t)$, when $\mathcal{F}(t)$ is essentially nonnegative with $t \in[0,1]$.

\section{AN ALGORITHM FOR CALCULATING THE DOMINANT EIGENVALUE}

Let $\mathcal{A}$ be an essentially nonnegative tensor of order $m$ and dimension $n$. In this section, we propose an algorithm to calculate the dominant eigenvalue of an essentially nonnegative tensor. This algorithm is a modification of the Ng-Qi-Zhou algorithm given in [12]. By Lemma 2.2 and Theorem 2.4, we modify the Ng-Qi-Zhou algorithm such that for any essentially nonnegative tensor, the sequence generated by the modified algorithm always converges to its dominant eigenvalue.

Define two functions from int $(P)$ to $P$ :

$$
F(x):=\min _{x_{i} \neq 0} \frac{\left(\mathcal{W} x^{m-1}\right)_{i}}{x_{i}^{m-1}}, \quad G(x):=\max _{x_{i} \neq 0} \frac{\left(\mathcal{W} x^{m-1}\right)_{i}}{x_{i}^{m-1}},
$$

where $\mathcal{W}$ is an irreducible nonnegative tensor. The details of the modified algorithm are given as follows.

\section{Algorithm 5.1}

Step 0. Given a sufficiently small number $\varepsilon>0$, let

$$
\mathcal{W}=\mathcal{A}+\alpha \mathcal{I}+\mathcal{E}
$$

where

$$
\alpha=\max _{1 \leqslant i \leqslant n}\left|A_{i \ldots i}\right|+1,
$$

and $\mathcal{E}$ is the tensor with every entry being $\varepsilon$. Choose any $x^{(0)} \in \operatorname{int}(P)$. Set $y^{(0)}=$ $\mathcal{W}\left(x^{(0)}\right)^{m-1}$ and $k:=0$.

Step 1. Compute

$$
x^{(k+1)}=\frac{\left(y^{(k)}\right)^{\left[\frac{1}{m-1}\right]}}{\left\|\left(y^{(k)}\right)^{\left[\frac{1}{m-1}\right]}\right\|}, \quad y^{(k+1)}=\mathcal{B}\left(x^{(k+1)}\right)^{m-1} .
$$

According to (13), compute $F\left(x^{(k+1)}\right)$ and $G\left(x^{(k+1)}\right)$.

Step 2. If $G\left(x^{(k+1)}\right)-F\left(x^{(k+1)}\right)<\varepsilon$, stop. Output $\varepsilon$-approximation of the dominant eigenvalue of $\mathcal{A}$ :

$$
\lambda^{(k+1)}=\frac{1}{2}\left(G\left(x^{(k+1)}\right)+F\left(x^{(k+1)}\right)\right)-\alpha,
$$

and the corresponding eigenvector $x^{(k+1)}$. Otherwise, set $k:=k+1$ and go to step 1 . 
Clearly, the tensor $\mathcal{W}$ defined by (14) is positive, and hence it is primitive [17, Corollary 3.7]. By Theorems 2.1 and 2.2, Algorithm 5.1 is well defined. As an immediate consequence of Lemma 2.2, Theorem 2.4, and Theorem 5.3 in [17], we have the following convergence theorem.

Theorem 5.1

Let $\mathcal{A}$ be an essentially nonnegative tensor of order $m$ and dimensional $n$, and let $\mathcal{W}$ be defined by (14) where $\varepsilon$ is a sufficiently small number. Then the sequences $\left\{F\left(x^{(k)}\right)\right\}$ and $\left\{G\left(x^{(k)}\right)\right\}$, generated by Algorithm 5.1, converge to $\lambda_{\varepsilon}$, where $\lambda_{\varepsilon}$ is the unique positive eigenvalue of $\mathcal{W}$. Moreover, the sequence $\left\{x^{(k)}\right\}$ converges to $x_{\varepsilon}^{*}$ and $x_{\varepsilon}^{*}$ is a positive eigenvector of $\mathcal{W}$ corresponding to the largest eigenvalue $\lambda_{\varepsilon}$. Furthermore,

$$
\lim _{\varepsilon \rightarrow 0} \lambda_{\varepsilon}=\lambda^{*}, \quad \lim _{\varepsilon \rightarrow 0} x_{\varepsilon}^{*}=x^{*},
$$

where $\lambda^{*}$ is the spectral radius of $\mathcal{A}+\alpha \mathcal{I}$ and $x^{*}$ is the corresponding eigenvector. In particular, the dominant eigenvalue of $\mathcal{A}$ is $\lambda(\mathcal{A})=\lambda^{*}-\alpha$, and $x^{*}$ is also the eigenvector corresponding to $\lambda(\mathcal{A})$.

Proof

It follows from (14) that $\mathcal{W}$ is positive, and hence it is irreducible. Therefore, for any nonzero $x \in P$, we have $\mathcal{W} x^{m-1} \in \operatorname{int}(P)$, which shows that the tensor $\mathcal{W}$ is primitive. Hence, by Theorem 5.3 in [17],

$$
\lim _{k \rightarrow \infty} F\left(x^{(k)}\right)=\lim _{k \rightarrow \infty}\left\{G\left(x^{(k)}\right)=\lambda_{\varepsilon}, \quad \lim _{k \rightarrow \infty} x^{(k)}=x_{\varepsilon}^{*} .\right.
$$

Therefore, $\lambda_{\varepsilon}-\alpha$ is an $\varepsilon$-approximation of the dominant eigenvalue of $\mathcal{A}$ from Theorem 2.4. Furthermore, it follows from Lemma 2.2 that

$$
\lim _{\varepsilon \rightarrow 0} \lambda_{\varepsilon}=\lambda^{*}, \quad \lim _{\varepsilon \rightarrow 0} x_{\varepsilon}^{*}=x^{*} .
$$

It is easy to see that $\lambda^{*}-\alpha$ is the dominant eigenvalue of $\mathcal{A}$ with corresponding eigenvector $x^{*}$.

The preceding theorem shows that the convergence of Algorithm 5.1 is established for any essentially nonnegative tensor without the irreducible and primitive assumption. In order to show the effectiveness of Algorithm 5.1, we used MATLAB 7.4 (MathWorks, Natick, MA) to test it on the following seven examples. The last four examples are large-scale numerical examples.

Example 5.1

Consider the three-order three-dimensional essentially nonnegative tensor

$$
\mathcal{A}=[A(1,:,:), A(2,:,:), A(3,:,:)],
$$

where

$$
\begin{aligned}
& A(:,:, 1)=\left(\begin{array}{rrr}
-1.51 & 8.35 & 1.03 \\
4.04 & 3.72 & 1.45 \\
6.71 & 6.43 & 1.35
\end{array}\right) \\
& A(:,:, 2)=\left(\begin{array}{rrr}
9.02 & 0.78 & 6.89 \\
9.71 & -5.32 & 1.85 \\
2.09 & 4.17 & 2.98
\end{array}\right) \\
& A(:,:, 3)=\left(\begin{array}{rrr}
9.55 & 1.57 & 6.91 \\
5.63 & 5.55 & 1.43 \\
5.76 & 8.29 & -0.15
\end{array}\right) .
\end{aligned}
$$

Example 5.2

Let a three-order three-dimensional tensor $\mathcal{A} 2$ be defined by $A_{133}=A_{233}=A_{311}=A_{322}=1$, $A_{111}=A_{222}=-1$, and zero otherwise. 
Example 5.3

Let a three-order four-dimensional tensor $\mathcal{A}$ be defined by $A_{111}=A_{222}=A_{333}=A_{444}=-1$, $A_{112}=A_{114}=A_{121}=A_{131}=A_{212}=A_{332}=A_{443}=1$, and zero otherwise.

Example 5.4

Let a three-order 500-dimensional tensor $\mathcal{A}$ be defined by $A_{1 j j}=1$ for $j \neq 1, A_{j 11}=1$ for $j \neq 1$, $A_{111}=-1, A_{222}=20$, and zero otherwise.

Example 5.5

Let a four-order 100-dimensional tensor $\mathcal{A}$ be defined by $A_{1 j j j}=1$ for $j \neq 1, A_{j 111}=1$ for $j \neq 1$, $A_{1111}=-1, A_{2222}=20$, and zero otherwise.

Example 5.6

Let $\mathcal{A}$ be a randomly generated three-order 200-dimensional tensor.

Example 5.7

Let $\mathcal{A}$ be a randomly generated three-order 50-dimensional tensor.

Clearly, the essentially nonnegative tensors defined in Examples 5.1 and 5.2 are irreducible, whereas the essentially nonnegative tensors defined in Examples 5.3-5.5 are reducible. The tensors defined in Examples 5.6 and 5.7 are randomly generated nonnegative tensors. The tensors defined in Examples 5.4 and 5.5 are sparse tensors.

We take $\varepsilon=10^{-9}$ and terminate our iteration when one of the conditions $G\left(x^{(k)}\right)-F\left(x^{(k)}\right) \leqslant$ $10^{-9}$ and $k \geqslant 100$ is satisfied. Algorithm 5.1 produces the dominant eigenvalue $\lambda(\mathcal{A})=36.2757$ with eigenvector $x^{*}=(1.0000 ; 0.8351 ; 0.9415)$ for Example 5.1, the dominant eigenvalue $\lambda(\mathcal{A})=$ 1 with eigenvector $x^{*}=(0.5000 ; 0.5000 ; 1.000)$ for Example 5.2, and the dominant eigenvalue $\lambda(\mathcal{A})=0.8225$ with eigenvector $x^{*}=(1.0000 ; 0.7408 ; 0.9714 ; 0.5330)$ for Example 5.3. For the large-scale tensors in the last four examples, we just list their dominant eigenvalues. Algorithm 5.1 produces the dominant eigenvalue $\lambda(\mathcal{A})=25.8107$ for Example 5.4, the dominant eigenvalue $\lambda(\mathcal{A})=8.9499$ for Example 5.5, the dominant eigenvalue $\lambda(\mathcal{A})=1.9995 \mathrm{e} 4$ for Example 5.6, and the dominant eigenvalue $\lambda(\mathcal{A})=6.2462 \mathrm{e} 4$ for Example 5.7,

The details of numerical results are reported in Tables I and II. We list the output details at each iteration for Example 5.1 in Table I. We also report the number of iterations (No.Iter), the elapsed

Table I. Detailed output of Algorithm 5.1 for Example 5.1.

\begin{tabular}{cccccc}
\hline$k$ & $\underline{\lambda}^{(k)}$ & $\bar{\lambda}^{(k)}$ & $\lambda^{(k)}$ & $\bar{\lambda}^{(k)}-\underline{\lambda}^{(k)}$ & $\Delta^{(k)}$ \\
\hline 1 & 35.9969 & 36.5635 & 36.2802 & 0.5666 & 0.2833 \\
2 & 36.2554 & 36.3030 & 36.2792 & 0.0476 & 0.0211 \\
3 & 36.2747 & 36.2776 & 36.2762 & 0.0030 & 0.0015 \\
4 & 36.2757 & 36.2758 & 36.2757 & $9.1725 \mathrm{e}-5$ & $4.5870 \mathrm{e}-5$ \\
5 & 36.2757 & 36.2757 & 36.2757 & $6.7568 \mathrm{e}-6$ & $2.9868 \mathrm{e}-6$ \\
6 & 36.2757 & 36.2757 & 36.2757 & $4.6425 \mathrm{e}-7$ & $1.4348 \mathrm{e}-7$ \\
7 & 36.2757 & 36.2757 & 36.2757 & $1.9041 \mathrm{e}-8$ & $8.1036 \mathrm{e}-9$ \\
\hline 8 & 36.2757 & 36.2757 & 36.2757 & $8.8998 \mathrm{e}-10$ & \\
\hline
\end{tabular}

Table II. Output of Algorithm 5.1 for Examples 5.1-5.7.

\begin{tabular}{lccccccc}
\hline Example & No.Iter & CPU $(\mathrm{s})$ & $\underline{\lambda}^{(k)}$ & $\bar{\lambda}^{(k)}$ & $\lambda^{(k)}$ & $\bar{\lambda}^{(k)}-\underline{\lambda}^{(k)}$ & $\Delta^{(k)}$ \\
\hline 5.1 & 8 & 0.013 & 36.2757 & 36.2757 & 36.2757 & $8.8998 \mathrm{e}-10$ & $8.1036 \mathrm{e}-9$ \\
5.2 & 31 & 0.035 & 1.0000 & 1.0000 & 1.0000 & $9.6831 \mathrm{e}-10$ & $4.1210 \mathrm{e}-9$ \\
5.3 & 37 & 0.078 & 0.8225 & 0.8225 & 0.8225 & $7.3324 \mathrm{e}-10$ & $1.0635 \mathrm{e}-8$ \\
5.4 & 39 & 931 & 25.8107 & 25.8107 & 25.8107 & $7.2051 \mathrm{e}-9$ & $3.9024 \mathrm{e}-9$ \\
5.5 & 21 & 647 & 8.9499 & 8.9499 & 8.9499 & $3.5831 \mathrm{e}-9$ & $2.5078 \mathrm{e}-9$ \\
5.6 & 4 & 3.9 & $1.9995 \mathrm{e} 4$ & $1.9995 \mathrm{e} 4$ & $1.9995 \mathrm{e} 4$ & $4.4001 \mathrm{e}-9$ & $1.3502 \mathrm{e}-9$ \\
5.7 & 4 & 7.3 & $6.2462 \mathrm{e} 4$ & $6.2462 \mathrm{e} 4$ & $6.2462 \mathrm{e} 4$ & $2.3059 \mathrm{e}-9$ & $4.1502 \mathrm{e}-9$ \\
\hline
\end{tabular}


CPU time (CPU (s)), the lower bound $\underline{\lambda}^{(k)}=F\left(x^{(k)}\right)-\alpha$ and the upper bound $\bar{\lambda}^{(k)}=G\left(x^{(k)}\right)-\alpha$

for $k \geqslant 1$, the error $\Delta^{(k)}=\left\|\mathcal{A}\left(x^{(k)}\right)^{m-1}-\lambda^{(k)}\left(x^{(k)}\right)^{[m-1]}\right\|_{\infty}$, and the approximation $\lambda^{(k)}$ defined by (15) of the dominant eigenvalue in Tables I and II.

From Tables I and II, we see that the sequence generated by Algorithm 5.1 converges to the dominant eigenvalue of the essentially nonnegative tensor without irreducibility. Algorithm 5.1 is promising for calculating the dominant eigenvalues of the seven examples. For the sparse tensors in Examples 5.4 and 5.5, the elapsed CPU times are longer because they need more iterations. Algorithm 5.1 can solve the non-sparse tensor in $20 \mathrm{~s}$ with the number of entries less than 100 million. For sparse tensors, Algorithm 5.1 is slow. Note that an internet link is made available to MATLAB codes of Algorithm 5.1 in the web http://www.polyu.edu.hk/ama/staff/new/ qilq/TensorComp.htm

\section{AN APPLICATION AND SOME CONCLUSIONS}

In this paper, we have introduced the concepts of essentially nonnegative tensors, which is closely related to nonnegative tensors. The main contribution is the convexity and log convexity of the dominant eigenvalue of an essentially nonnegative tensor, and hence the same for the spectral radius of a nonnegative tensor. We also have proposed an algorithm for calculating the dominant eigenvalue, and convergence analysis has been established for any essentially nonnegative tensor without the assumptions of irreducibility and primitiveness.

As an application, we find that the convexity of the maximal eigenvalue function plays an important role in the trace-preserving problem, which arises in signal processing system [26,27]. The trace-preserving problem is to determine $\mu(\mathcal{A})=\min \left\{\lambda\left(\mathcal{A}+\mathcal{D}_{u}\right): e^{\mathrm{T}} u=0\right\}$ and to find a vector $u=\left(u_{1}, \ldots, u_{n}\right)^{\mathrm{T}}$ that achieves this minimum, where $\mathcal{A}$ is an essentially nonnegative tensor and $\mathcal{D}_{u}$ is a diagonal tensor with $u_{1}, \ldots, u_{n}$ as the diagonal entries. By Theorem 3.1 and Corollary 3.1, this problem is a convex problem. Motivated by the idea in [26], we guess that the semismoothness of the dominant eigenvalue function also holds, and then we may propose a Newton-type algorithm to solve the trace-preserving problem. This is a topic in the future research.

\section{ACKNOWLEDGEMENTS}

The authors would like to thank the editor and the anonymous referees for their constructive comments and suggestions, which lead to a significantly improved version of the paper.

Liping Zhang's work was supported by the National Natural Science Foundation of China (grant no. 11271221). Liqun Qi's work was supported by the Hong Kong Research Grant Council (grant nos. PolyU 501909, 502510, 502111, and 501212). Ziyan Luo's work was supported by the National Basic Research Program of China (2010CB732501) and the National Natural Science Foundation of China (11101248).

\section{REFERENCES}

1. Van Loan CF. Future directions in tensor-based computation and modeling. NSF Workshop Report in Arlington, Virginia, USA, 2009. (Available from: http://www.cs.cornell.edu/cv/TenWork/Home.htm).

2. Qi L. The spectral theory of tensors (rough version), arXiv:1201.3424 [math SP] 17 Jan 2012. (Available from: http://www.polyu.edu.hk/ama/staff/new/qilq/TensorComp.htm).

3. Qi L. Eigenvalues of a real supersymmetric tensor. Journal of Symbolic Computation 2005; 40:1302-1324.

4. Lim LH. Singular values and eigenvalues of tensors: a variational approach. In Proceedings of the IEEE International Workshop on Computational Advances in Multi-sensor Adaptive Processing (CAMSAP'05), Vol. 1. IEEE Computer Society Press: Piscataway, NJ, 2005; 129-132.

5. Qi L, Dai HH, Han D. Conditions for strong ellipticity and $M$-eigenvalues. Frontiers of Mathematics in China 2009; 4:349-364.

6. Qi L, Wang Y, Wu EX. D-eigenvalues of diffusion kurtosis tensor. Journal of Computational and Applied Mathematics 2008; 221:150-157.

7. Chang KC, Pearson K, Zhang T. On eigenvalue problems of real symmetric tensors. Journal of Mathematics Analysis and Applications 2009; 350:416-422.

8. Cartwright D, Sturmfels B. The number of eigenvalues of a tensor. Linear Algebra and Applications 2013; 438:942-952. 
9. Bulò SR, Pelillo M. A generalization of the Motzkin-Straus theorem to hypergraphs. Optimization Letters 2009; 3:187-295.

10. Bulò SR, Pelillo M. New bounds on the clique number of graphs based on spectral hypergraph theory. In Learning and Intelligent Optimization, Stützle T (ed.). Springer Verlag: Berlin, 2009; 45-48.

11. Hu S, Qi L. Algebraic connectivity of an even uniform hypergraph. Journal of Combinatorial Optimization 2012; 24:564-579.

12. Ng M, Qi L, Zhou G. Finding the largest eigenvalue of a nonnegative tensor. SIAM Journal on Matrix Analysis and Applications 2009; 31:1090-1099.

13. Chang KC, Pearson K, Zhang T. Perron Frobenius theorem for nonnegative tensors. Communications in Mathematical Sciences 2008; 6:507-520.

14. Friedland S, Gaubert S, Han L. Perron-Frobenius theorem for nonnegative multilinear forms and extensions. Linear Algebra and Its Applications 2013; 438:738-749.

15. Yang YN, Yang QZ. Further results for Perron-Frobenius theorem for nonnegative tensors. SIAM Journal on Matrix Analysis and Applications 2010; 31:2517-2530.

16. Collatz L. Einschliessungssatz für die charakteristischen Zahlen von Matrizen. Mathematik Zeitschrift 1942; 48:221-226.

17. Chang KC, Pearson K, Zhang T. Primitivity, the convergence of the NZQ method, and the largest eigenvalue for nonnegative tensors. SIAM Journal on Matrix Analysis and Applications 2011; 32:806-819.

18. Zhang LP, Qi L. Linear convergence of an algorithm for computing the largest eigenvalue of a nonnegative tensor. Numerical Linear Algebra with Applications 2012; 19:830-841.

19. Liu Y, Zhou G, Ibrahim NF. An always convergent algorithm for the largest eigenvalue of an irreducible nonnegative tensor. Journal of Computational and Applied Mathematics 2010; 235:286-292.

20. Zhang LP, Qi L, Xu Y. Linear convergence of the LZI algorithm for weakly positive tensors. Journal of Computational Mathematics 2012; 30:24-33.

21. Friedland S. Convex spectral functions. Linear and Multilinear Algebra 1981; 9:299-316.

22. Horn R, Johnson CH. Matrix Analysis. Cambridge University Press: Cambridge, 1996.

23. Nussbaum RD. Convexity and log convexity for the spectral radius. Linear Algebra and Its Applications 1986; 73:59-122.

24. Roth R. On the eigenvectors belonging to the minimum eigenvalue of an essentially nonnegative symmetric matrix with bipartite graph. Linear Algebra and its Applications 1989; 118:1-10.

25. Harker PT. Derivatives of the Perron root of a positive reciprocal matrix: with application to the analytic hierarchy process. Applied Mathematics and Application 1987; 22:217-232.

26. Li G, Qi L, Yu G. Semismoothness of the maximum eigenvalue function of a symmetric tensor and its application. Linear Algebra and Its Applications 2013; 438:813-833.

27. Johnson CR, Stanford DP. Dominant eigenvalues under trace-preserving diagonal perturbations. Linear Algebra and Its Applications 1994; 212:415-435. 